\title{
Absolute and Relative Time-Consistent Revealed Preferences*
}

\author{
Thomas Demuynck ${ }^{\dagger}$ \\ January 7, 2008
}

\begin{abstract}
We introduce an Absolute (Relative) Time-consistent Axiom of Revealed Preference which characterizes the consistency of a choice function with the property of absolute (relative) time-consistency and impatience. The axiom requires that the absolute (relative) time-consistent and impatient closure of the revealed preference relation does not conflict with the strict revealed preference relation.
\end{abstract}

Keywords: Time-consistency; Revealed Preference, Choice Function.

\section{Introduction}

Consider a preference relation over a universal set of alternatives, $X$. In order to pick a best element out of every two element choice set (subset of $X$ ), it is necessary that the preference relation is complete. If we want to pick a best element out of any larger choice set, we must also impose transitivity. Additional requirements on the preference relation normally require additional structure on the universal set. This paper investigates the implication of requirements frequently encountered in intertemporal settings.

If we want to impose properties relating to the time-instance at which the various alternatives are consumed, we can represent the universal set of alternatives as $X \times T$, where $X$ is a set of alternatives and $T$ is a set of time instances. A preference relation is then a transitive and complete binary relation on this extended set.

Given transitivity and completeness, this research discusses the implication of three additional intertemporal properties.

For the first property, consider two time-instances $t$ and $v$, with instance $t$ before instance $v$. We say that a preference relation is 'impatient' if for any alternative $x$, the bundle $(x, t)$ is at least as good as the bundle $(x, v)$. This condition seems intuitive, at least if goods are nonperishable and storage is costless. Since this is such a basic requirement in an intertemporal context, we maintain it throughout, and combine it successively with each of the two following properties.

For the next property, consider two bundles $(x, t)$ and $(y, v)$ and assume that $(x, t)$ is at least as good as $(y, v)$. After a certain amount of time $s \leq t, v$, the individual is asked to

\footnotetext{
${ }^{*}$ We acknowledge financial support from the Research Foundation-Flanders (FWO-Vlaanderen) and from the Inter-university Attraction Poles Programme - Belgian Science Policy [Contract NO.P6/07]. The usual disclaimer applies.

${ }^{\dagger}$ Sherppa, Ghent University, Hoveniersberg 24, B-9000 Gent, Belgium.
} 
reconsider the two bundles, which have now become $(x, t-s)$ and $(y, v-s)$. It would seem natural to require that individuals do not change their minds (preferences) as time goes by. Therefore, we should have that $(x, t-s)$ is at least as good as $(y, v-s)$. This property is called 'absolute time-consistency' and it is one of the key assumptions in the characterization of the exponential discounted utility model (see for example Fishburn and Rubinstein [1982]).

The final property is a variant on the second property and is called 'relative time-consistency'. It states, contrary to absolute time-consistency, that preferences over alternatives depend on the relative time differences between the two consumption periods instead of the absolute difference. In particular, if $(x, t)$ is at least as good as $(y, v)$, then for any strict positive real number $s$, the bundle $(x, t . s)$ is at least as good as the bundle $(y, v . s)$. This property is a key property for the characterization of the hyperbolic time preference model as in Harvey [1986].

This paper presents two revealed preference axioms which guarantees the existence of an absolute or relative time-consistent and impatient preference relation rationalizing a given choice function. The revealed preference axioms, which we call the absolute and relative time-consistent axiom of revealed preference (ATARP and RTARP), state that the absolute (relative) time-consistent and impatient closure of the revealed preference relation does not conflict with the strict revealed preference relation. The computation of these closures requires the computation of a transitive closure and a simple algebraic expression. In this perspective, it is computationally only slightly more demanding than computing the transitive closure.

Most research, investigating the plausibility of the absolute or relative time-consistency (and impatience) assumption start from a specific functional form for the (instantaneous) utility function, and try to fit the model to the observed data (e.g. Eisenhauer and Ventura [2006], Angeletos et al. [2001], for a good overview see Frederick et al. [2002]). Our revealed preference approach has a clear advantage over this approach (Sen [1971], Richter [1966])): the axioms do not depend on a particular functional form of the preference relation. In fact, the preference relation does not even need to have a functional form ${ }^{1}$.

Section 2 presents notation and provides us with the characterization results and section 3 presents the proofs.

\section{Notation and results}

Let $X$ be the universal set of alternatives and let $T=\mathbb{R}^{+}$denote the universal set of time instances. The present is set at time equal to 0 . We also define the set $T_{0}=T-\{0\}$. An element $(x, t)$ of $X \times T$ denotes the consumption of alternative $x$ at time $t$. A binary relation $R$ is a subset of $(X \times T)^{2}$. Define $R^{-1}$ as $(x, y) \in R^{-1}$ if and only if $(y, x) \in R$. The asymmetric part $P(R)$ of the relation $R$ is given by $R-R^{-1}$, the symmetric part $I(R)$ of $R$ is given by $R \cap R^{-1}$ and the non-comparable part $N(R)$ is given by $(X \times T)^{2}-\left(R \cup R^{-1}\right)$. A relation $R$ is transitive if for all $x, y, z \in X$ and $t, v, w \in T:((x, t),(y, v)) \in R$ and $((y, v),(z, w)) \in R$ implies $((x, t),(z, w)) \in R$. The relation $R$ is complete if for all $x, y \in X$ and $t, v \in T$ : $((x, t),(y, v)) \in R \cup R^{-1}$. A complete and transitive relation is called an ordering.

\footnotetext{
${ }^{1}$ See also remark 3 in section 2.
} 
Definition 1 (Impatience). A relation $R$ on $X \times T$ is impatient if for all $x$ in $X$ and $t, v$ in $T$ with $t \leq v$ :

$$
((x, t),(x, v)) \in R .
$$

Any, not necessarily transitive and complete, relation $R$ on $X \times T$ can be enlarged to an impatient relation, $R^{\prime}$, given by:

$$
R^{\prime}=R \cup\left\{((x, t),(x, v)) \in(X \times T)^{2} \mid t \leq v\right\} .
$$

Notice that in the definition of $R^{\prime}$ we keep the alternative $x$ the same in both bundles. Given a relation $R$, we call $R^{\prime}$ the impatient closure of the relation $R$. It is the smallest impatient relation that contains $R$.

Definition 2 (absolute time-consistency). The relation $R$ is said to be absolute time-consistent if for all $x, y \in X ; t, v \in T$ and $s \leq t, v$ :

$$
((x, t),(y, v)) \in R \leftrightarrow((x, t-s),(y, v-s)) \in R .
$$

Definition 3 (relative time-consistency). The relation $R$ is said to be relative time-consistent if for all $x, y \in X ; t, v \in T_{0}$ and $s>0$ :

$$
((x, t),(y, v)) \in R \leftrightarrow((x, t . s),(y, v . s)) \in R .
$$

Notice that for relative time-consistency, we restrict the time domain to $T_{0}$. The reason for this will become clear when we define the relative time-consistent closure.

We already introduced the impatient closure of a relation $R$ as the smallest impatient relation that contains $R$. In a similar fashion we define the absolute time-consistent closure of the relation $R$ as the smallest transitive and absolute time-consistent relation containing $R$. Before we give the formal definition, let us start with an example. Let $R$ be an arbitrary relation and let us introduce $\bar{R}$ as its absolute time-consistent closure. Consider elements $x, y$ and $z$ in $X$ and assume that

$$
((x, 3),(y, 2)) \in R \text { and }((y, 6),(z, 4)) \in R \text {. }
$$

We know that $\bar{R}$ contains $R$ and that it satisfies absolute-time consistency, so we can add an equal amount, 4 , to 3 and 2 to get:

$$
((x, 7),(y, 6)) \in \bar{R} \text { and }((y, 6),(z, 4)) \in \bar{R} .
$$

As $\bar{R}$ is also required to satisfy transitivity, we have that $((x, 7),(z, 4)) \in \bar{R}$. Following the calculations through, we see that 7 was obtained as $3+(6-2)$, hence we can write $7-4$ as $(3-2)+(6-4)$. Also, notice that by absolute time-consistency of $\bar{R}$, only the absolute difference between 7 and 4 really matters. As such, we derive that for all $t$ and $v$ in $T$ for which $t-v=7-4=(3-2)+(6-4)$ :

$$
((x, t),(z, v)) \in \bar{R} \text {. }
$$

It is easy to see that this example does not rely on the specific values of $3,2,6$ and 4 . Hence, we can substitute $3=t_{1}, 2=v_{1}, 6=t_{2}$ and $4=v_{2}$, and derive that:

$$
\left(\left(x, t_{1}\right),\left(y, v_{1}\right)\right) \in R \text { and }\left(\left(y, t_{2}\right),\left(z, v_{2}\right)\right) \in R \rightarrow((x, t),(z, v)) \in \bar{R},
$$


where $t$ and $v$ satisfy $t-v=\left(t_{1}-v_{1}\right)+\left(t_{2}-v_{2}\right)$.

This example only relates to two element subsets of $R$. The generalization to all finite subsets leads to the following definition ${ }^{2}$.

Definition 4 (absolute time-consistent closure). The absolute time-consistent closure $\bar{R}$ of $R$ is defined as: $((x, t),(y, v)) \in \bar{R}$ if there is a sequence $x=x_{1}, \ldots, x_{n}=y$ of elements in $X$ and sequences $t_{1}, \ldots, t_{n-1}$ and $v_{1}, \ldots, v_{n-1}$ of elements in $T$ such that for all $i=1, \ldots, n-1$ :

$$
\left(\left(x_{i}, t_{i}\right),\left(x_{i+1}, v_{i}\right)\right) \in R
$$

and

$$
t-v=\sum_{i=1}^{n-1}\left(t_{i}-v_{i}\right) .
$$

If we follow a similar reasoning for the property of relative time-consistency, we are led to the following definition:

Definition 5 (relative time-consistent closure). The relative time-consistent closure $\tilde{R}$ of $R$ is defined by: $((x, t),(y, v)) \in \tilde{R}$ if there is a sequence $x=x_{1}, \ldots, x_{n}=y$ of elements in $X$ and sequences $t_{1}, \ldots, t_{n-1}$ and $v_{1}, \ldots, v_{n-1}$ of elements in $T_{0}$ such that for all $i=1, \ldots, n-1$ :

$$
\left(\left(x_{i}, t_{i}\right),\left(x_{i+1}, v_{i}\right)\right) \in R
$$

and

$$
\frac{t}{v}=\prod_{i=1}^{n-1} \frac{t_{i}}{v_{i}}
$$

Consider an arbitrary relation $R$, and take its corresponding impatient closure $R^{\prime}$ (see definition 1). The absolute time-consistent closure of $R^{\prime}$ is then denoted by $\bar{R}^{\prime}$. Lemma 1 in the next section shows that $\bar{R}^{\prime}$ is the smallest transitive, absolute time-consistent and impatient relation containing $R$. As such, we also call it the absolute time-consistent and impatient closure of $R$. Likewise, we let $\tilde{R}^{\prime}$ be the relative time-consistent closure of $R^{\prime}$ and we call it the relative time-consistent and impatient closure of $R$.

Consider the set $\Lambda=2^{X \times T}-\{\emptyset\}$ which is the set of all non-empty choice sets. A choice function, $C$, is a function from a set $\Sigma \subseteq \Lambda$ to $\Lambda$ such that for all $A \in \Sigma: C(A) \subseteq A$. The set $C(A)$ is to be interpreted as the choices made from the set $A$.

A choice function $C$ is said to be rationalizable by an absolute time-consistent and impatient ordering if and only if there exist an absolute time-consistent and impatient ordering $R$ on $X \times T$ such that for all $A \in \Sigma$ :

$$
C(A)=\{(x, t) \in A \mid \forall(y, v) \in A:((x, t),(y, v)) \in R\} .
$$

In plain English: the choices made from $A$ are the ones that are top ranked according to the ordering $R$.

\footnotetext{
${ }^{2}$ The proof that the relation $\bar{R}$ in definition 4 coincides with the smallest transitive and absolute timeconsistent relation containing $R$ is almost identical to the proof of Lemma 1 in section 3 and is therefore omitted.
} 
To introduce the notion of relative time-consistent rationalizability, we adjust the set of choice sets to $\Lambda_{0}=2^{X \times T_{0}}$ and we introduce a choice function, $C_{0}$, as a function from a set $\Sigma_{0} \subseteq \Lambda_{0}$ to $\Lambda_{0}$ such that for all $A \in \Sigma_{0}: C_{0}(A) \subseteq A$.

Analogously, a choice function $C_{0}$ is rationalizable by a relative time-consistent and impatient ordering if and only if there exist a relative time-consistent and impatient ordering $R$ on $X \times T_{0}$ such that for all $A \in \Sigma_{0}$ :

$$
C_{0}(A)=\{(x, t) \in A \mid \forall(y, v) \in A:((x, t),(y, v)) \in R\} .
$$

Given a choice function, $C$, we can define the revealed preference relation $R_{v}$ as $((x, t),(y, v)) \in$ $R_{v}$ if and only if there is an $A \in \Sigma$ such that $(x, t) \in C(A)$ and $(y, v) \in A$. The strict revealed preference relation $P_{v}$ is defined as $((x, t),(y, v)) \in P_{v}$ if and only if there is an $A \in \Sigma$ such that $(x, t) \in C(A)$ and $(y, v) \in A-C(A)$. These definitions remains the same when we change from $\Sigma$ and $C$ to $\Sigma_{0}$ and $C_{0}$.

Consider a choice function $C$ and assume that $C$ is rationalizable by an absolute timeconsistent and impatient ordering $R$. The choice function, $C$, is only defined over the set $\Sigma$, so in general it is not possible to reconstruct the ordering $R$ from the observation of $C$. On the other hand, we do observe the relations $R_{v}$ and $P_{v}$. From the rationalizability of $C$, we know that $R_{v} \subseteq R$ and $P_{v} \subseteq P(R)$. The ordering $R$ is transitive, absolute time-consistent and impatient, hence it must include the absolute time-consistent and impatient closure of $R_{v}$, i.e. $\bar{R}_{v}^{\prime} \subseteq R$. Therefore, it must be that $\bar{R}_{v}^{\prime} \cap P_{v}^{-1}$ is empty. If not, we would have that $R \cap P(R)^{-1}$ is also non-empty and this would contradicts the definition of the asymmetric part. We call this property the Absolute Time-consistent Axiom of Revealed Preference.

Definition 6 (ATARP). A choice function $C$ satisfies the absolute time-consistent axiom of revealed preference if

$$
\bar{R}_{v}^{\prime} \cap P_{v}^{-1}=\emptyset .
$$

Similarly, we may define the Relative Time-consistent Axiom of Revealed Preference in the following way:

Definition 7 (RTARP). A choice function $C_{0}$ satisfies the relative time-consistent axiom of revealed preference if

$$
\tilde{R}_{v}^{\prime} \cap P_{v}^{-1}=\emptyset .
$$

As shown above, the ATARP (RTARP) is a necessary condition for a choice function to be rationalizable by an absolute (relative) time-consistent and impatient ordering. Fortunately, it turns out that it is also a sufficient condition. We state this in the following theorems. The proof is given in the next section.

Theorem 1. A choice function $C$ is rationalizable by an absolute time-consistent and impatient ordering if and only if it satisfies the ATARP.

Theorem 2. A choice function $C_{0}$ is rationalizable by a relative time-consistent and impatient ordering if and only if it satisfies the RTARP.

We conclude this section with a few remarks.

Remark 1. We assumed that $T=\mathbb{R}^{+}$, but we can easily change this to a smaller set $T^{\prime} \subset \mathbb{R}^{+}$, e.g. $T^{\prime}=\mathbb{N}$, and the theorems remains valid. To see this, notice that we can select the set $\Sigma$ 
to select only sets $A$ which have elements with time components in $T^{\prime}$. The theorems give rise to a rationalization $R^{*}$, which is defined over the entire set $X \times T$. However, the restriction of this $R^{*}$ to the set $X \times T^{\prime}$ will provide us with a rationalization on this smaller domain.

Remark 2. Sometimes, the definition of time-consistency is defined in term of sequences, $\left(x_{i}, t\right),\left(x_{i+1}, t+1\right) \ldots,\left(x_{i+n}, t+n\right)$, of consumption bundles instead of individual consumption bundles. In this sense, we can say that a relation $R$ is $n$ absolute time-consistent if for all $t, v \in \mathbb{N}, t, v \in \mathbb{Z}$ and $s \leq t, v$ :

$$
\left(\left(\left(x_{i}, t\right), \ldots,\left(x_{i+n}, t+n\right)\right),\left(\left(y_{i}, v\right), \ldots,\left(y_{i+n}, v+n\right)\right)\right) \in R
$$

if and only if:

$$
\left(\left(\left(x_{i}, t-s\right), \ldots,\left(x_{i+n}, t+n-s\right)\right),\left(\left(y_{i}, v-s\right) \ldots,\left(y_{i+n}, v+n-s\right)\right)\right) \in R .
$$

Let us take the set of alternatives to be $\tilde{X}$ and redefine our universal set $X$ as $X=\tilde{X}^{n}$. If we let $\left(x_{1}, \ldots, x_{n}, t\right)=(x, t) \in X \times T$ denote the bundle $\left(\left(x_{1}, t\right), \ldots,\left(x_{n}, t+n\right)\right)$ we see immediately that our definition of absolute time-consistency is the same as the definition of $n$ absolute time-consistency given above. As such, taking into account remark 1, the main result carries directly over to this alternative definition of time-consistency. An analogous result holds of course also for the property of relative time-consistency. Also, setting $X=\tilde{X}^{\infty}$, allows for infinite sequences.

Remark 3. The existence of an absolute (relative) time-consistent ordering on $X \times T$ does not specify anything on the specific functional form of this ordering. Moreover, it may even be the case, that no functional (real valued) representation exists. As an example, consider ordering $R$ where:

$$
((x, t),(y, v)) \in R \leftrightarrow t<v \text { or }(t=v \text { and }(x, y) \in Q),
$$

with $Q$ an ordering on the set $X$. The ordering $R$ is absolute time-consistent, relative timeconsistent and impatient. On the other hand, $R$ is a lexicographic ordering and it is well known that such an ordering has no real valued representation (e.g. Durieu et al. [2006], Example 4 or Debreu [1954]).

\section{Proof of theorem 1}

We provide the proof for the case of rationalizability by an absolute time-consistent and impatient ordering (Theorem 1). The proof for Theorem 2 is completely analogous and is left to the reader.

Let $R$ be an arbitrary relation on $X \times T$ and consider the relation $\bar{R}^{\prime}$. We have the following result:

Lemma 1. For a relation $R$, the closure $\bar{R}^{\prime}$ is the smallest, transitive, absolute time-consistent and impatient relation containing $R$.

Proof. That $\bar{R}^{\prime}$ contains $R$ is obvious. As $\bar{R}^{\prime}$ also contains $R^{\prime}$, we have that $\bar{R}^{\prime}$ is impatient. To see that $\bar{R}^{\prime}$ is absolute time-consistent, assume that $((x, t),(y, v)) \in \bar{R}^{\prime}$ so that there is a sequence $x=x_{1}, \ldots, x_{n}=y$ in $X$ and sequences $t_{1}, \ldots, t_{n-1}$ and $v_{1}, \ldots, v_{n-1}$ in $T$ such that for all $i=1, \ldots, n-1$ :

$$
\left(\left(x_{i}, t_{i}\right),\left(x_{i+1}, v_{i}\right)\right) \in R^{\prime}
$$


and

$$
t-v=\sum_{i=1}^{n-1}\left(t_{i}-v_{i}\right)
$$

Immediately, we see that for any $s \leq t, v$ :

$$
(t-s)-(v-s)=\sum_{i=1}^{n-1}\left(t_{i}-v_{i}\right)
$$

This implies that: $((x, t-s),(y, v-s)) \in \bar{R}^{\prime}$, hence $\bar{R}^{\prime}$ is absolute time-consistent.

To prove that $\bar{R}^{\prime}$ is transitive, let $((x, t),(y, v)) \in \bar{R}^{\prime}$ and $((y, v),(z, w)) \in \bar{R}^{\prime}$ so that there are sequences $x=x_{1}, \ldots, x_{n}=y$ and $y=y_{1}, \ldots, y_{m}=z$ in $X$ and sequences $t_{1}, \ldots, t_{n-1} ; v_{1}, \ldots, v_{n-1}$; $s_{1}, \ldots, s_{m-1}$ and $w_{1}, \ldots, w_{m-1}$ in $T$ such that for all $i=1, \ldots, n-1$ and $j=1, \ldots, m-1$ :

$$
\begin{gathered}
\left(\left(x_{i}, t_{i}\right),\left(x_{i+1}, v_{i}\right)\right) \in R^{\prime}, \\
\left(\left(y_{j}, s_{j}\right),\left(y_{j+1}, w_{j}\right)\right) \in R^{\prime}, \\
t-v=\sum_{i=1}^{n-1}\left(t_{i}-v_{i}\right)
\end{gathered}
$$

and

$$
v-w=\sum_{j=1}^{m-1}\left(s_{j}-w_{j}\right) .
$$

Consider the compound sequence $x=x_{1}, \ldots, x_{n}, y_{2}, \ldots, y_{m}=z$ in $X$ and the compound sequences $t_{1}, \ldots, t_{n-1}, s_{1}, \ldots, s_{m-1}$ and $v_{1}, \ldots, v_{n-1}, w_{1}, \ldots, w_{m-1}$ in $T$. As:

$$
t-v+v-w=t-w=\sum_{i=1}^{n-1}\left(t_{i}-v_{i}\right)+\sum_{j=1}^{m-1}\left(s_{j}-w_{j}\right),
$$

we can conclude that $((x, t),(z, w)) \in \bar{R}^{\prime}$, hence $\bar{R}^{\prime}$ is transitive.

We are left to show that $\bar{R}^{\prime}$ is the smallest transitive, absolute time-consistent and impatient relation containing $R$. Assume on the contrary that there is a relation $R^{*}$ which contains $R$ and is transitive, absolute time-consistent and impatient and that there exist elements $x, y \in X$ and time instances $t, v \in T$, such that $((x, t),(y, v)) \in \bar{R}^{\prime}$ and $((x, t),(y, v)) \notin R^{*}$. Consequently, there is a sequence $x=x_{1}, \ldots, x_{n}=y$ in $X$ and sequences $t_{1}, \ldots, t_{n-1}$ and $v_{1}, \ldots, v_{n-1}$ in $T$ such that for all $i=1, \ldots, n-1$ :

$$
\left(\left(x_{i}, t_{i}\right),\left(x_{i+1}, v_{i}\right)\right) \in R^{\prime}
$$

and

$$
t-v=\sum_{i=1}^{n-1}\left(t_{i}-v_{i}\right)
$$

We show that $((x, t),(y, v)) \in R^{*}$ by induction on $n$. For $n=2$, we have that:

$$
\left(\left(x, t_{1}\right),\left(y, v_{1}\right)\right) \in R^{\prime}
$$


and

$$
t-v=t_{1}-v_{1} .
$$

As $R^{*}$ is impatient, we derive that $\left(\left(x, t_{1}\right),\left(y, v_{1}\right)\right) \in R^{*}$. Also, we have that $t_{1}-t=v_{1}-v \leq t_{l}$ hence setting $s=t_{1}-t$ and applying absolute time-consistency of $R^{*}$, we obtain:

$$
((x, t),(y, v)) \in R^{*} .
$$

For the induction step, suppose that $((x, t),(y, v)) \in R^{*}$ whenever $\left((x, t),(y, v) \in \bar{R}^{\prime}\right.$ for all $n \leq l$ and consider the case where $n=l+1$. This implies that there is a sequence $x=x_{1}, \ldots, x_{l}, x_{l+1}=y$ and sequences $t_{1}, \ldots, t_{l}$ and $v_{1}, \ldots, v_{l}$ such that for all $i=1, \ldots, l$ :

$$
\left(\left(x_{i}, t_{i}\right),\left(x_{i+1}, v_{i}\right)\right) \in R^{\prime}
$$

and

$$
t-v=\sum_{i=1}^{l}\left(t_{i}-v_{i}\right) .
$$

Take two elements $t^{\prime}$ and $v^{\prime}$ from $T$ such that:

$$
t^{\prime}-v^{\prime}=\sum_{i=1}^{l-1}\left(t_{i}-v_{i}\right)
$$

Hence $t^{\prime}-v^{\prime}+t_{l}-v_{l}=t-v$. From the induction hypothesis, we have that $\left(\left(x, t^{\prime}\right),\left(x_{l}, v^{\prime}\right)\right) \in R^{*}$. Observe that also $\left(\left(x_{l}, t_{l}\right),\left(y, v_{l}\right)\right) \in R^{*}$. If $v^{\prime} \geq t_{l}$ put $s=t_{l}-v^{\prime}$ and from absolute timeconsistency, we have that: $\left(\left(x, t^{\prime}\right),\left(x_{l}, v^{\prime}\right)\right) \in R^{*}$ and $\left(\left(x_{l}, v^{\prime}\right),\left(y, v_{l}-t_{l}+v^{\prime}\right)\right) \in R^{*}$. From transitivity, we derive that $\left(\left(x, t^{\prime}\right),\left(y, t^{\prime}-t+v\right)\right) \in R^{*}$. Put $s=t^{\prime}-t$ and apply the absolute time-consistency of $R^{*}$. We derive that $((x, t),(y, v)) \in R^{*}$. The case where $v^{\prime} \leq t_{l}$ is solved similarly. Conclude that $((x, t),(y, v)) \in R^{*}$, a contradiction. Hence, $\bar{R}^{\prime}$ is indeed the smallest (transitive, absolute time-consistent and impatient) relation containing $R$.

Consider a relation $R$ on $X \times T$. We say that $R^{*}$ is an extension of $R$ if $R \subseteq R^{*}$ and $P(R) \subseteq P\left(R^{*}\right)$

Lemma 2. A relation $R$ has a time-consistent and impatient ordering extension $R^{*}$ if and only if:

$$
\bar{R}^{\prime} \cap P(R)^{-1}=\emptyset .
$$

Proof. The proof is similar to the proof of Szpilrajn's lemma (Szpilrajn [1930]) ${ }^{3}$, which states that every quasi-order (transitive and reflexive binary relation) has an ordering extension.

To see necessity assume that $R$ has an absolute time-consistent and impatient ordering extension, $R^{*}$, and assume on the contrary that there exist $((x, t),(y, v)) \in \bar{R}^{\prime} \cap P(R)^{-1}$. From lemma 1 , we have that $\bar{R}^{\prime}$ is the smallest absolute time-consistent, impatient and transitive relation containing $R$. As $R^{*}$ is also an absolute time-consistent, impatient and transitive relation, we must have that $\bar{R}^{\prime} \subseteq R^{*}$. This means that $((x, t),(y, v)) \in R^{*}$. Also, as $R^{*}$ is an extension of $R$, we must have that $((y, v),(x, t)) \in P\left(R^{*}\right)$, which is in contradiction with the definition of $P\left(R^{*}\right)$. Hence, $\bar{R}^{\prime} \cap P(R)^{-1}=\emptyset$.

\footnotetext{
${ }^{3}$ For further generalizations of Szpilrajn's lemma, see Suzumura [1976], Duggan [1999] and Donaldson and Weymark [1998] among others.
} 
For sufficiency, consider the set $\Omega$ of all extensions, $Q$, of $R$ for which $\bar{Q}^{\prime} \cap P(Q)^{-1}=\emptyset$. This set is non-empty as $R \in \Omega$. Let $\Omega^{\prime}$ be a chain in $\Omega$, i.e. for all $Q, S \in \Omega$ either $Q \subseteq S$ or $S \subseteq Q$. Consider the relation $B=\bigcup_{Q \in \Omega^{\prime}} Q$ and let us show that $B \in \Omega$. It is easy to see that $B$ is an extension of $R$. To see that $\bar{B}^{\prime} \cap P(B)^{-1}=\emptyset$, assume on the contrary that there exist elements $x, y \in X$ and $t, v \in T$ such that $((x, t),(y, v)) \in \bar{B}^{\prime} \cap P(B)^{-1}$. Then, there exist a sequence $x=x_{1}, \ldots, x_{n}=y$ of elements in $X$ and sequences $t_{1}, \ldots, t_{n-1}$ and $v_{1}, \ldots, v_{n-1}$ such that for all $i=1, \ldots, n-1$ :

$$
\left(\left(x_{i}, t_{i}\right),\left(x_{i+1}, v_{i}\right)\right) \in B
$$

and

$$
t-v=\sum_{i=1}^{n-1}\left(t_{i}-v_{i}\right) .
$$

From the construction of $B$, we see that there must be relations $Q_{1}, \ldots, Q_{n-1}$ in $\Omega^{\prime}$ such that for all $i \leq n-1,\left(\left(x_{i}, t_{i}\right),\left(x_{i+1}, v_{i}\right) \in Q_{i}\right.$. As all these relations are ranked by set inclusion, there must be a largest one, lets say $Q_{j}$. Also, from the definition of $B$, there must be a relation $Q_{0} \in \Omega^{\prime}$ such that $((y, v),(x, t)) \in Q_{0}$ and for all $Q \in \Omega^{\prime}$ we can not have that $((x, t),(y, v)) \in Q$. The relations $Q_{0}$ and $Q_{j}$ are ranked by set inclusion so either $Q_{j} \subseteq Q_{0}$ or $Q_{0} \subseteq Q_{j}$. In the first case, we have that $((x, t),(y, v)) \in \bar{Q}_{0}^{\prime} \cap P\left(Q_{0}\right)^{-1}$, contradicting the fact that $Q_{0} \in \Omega$. In the second case, we have that $((x, t),(y, v)) \in \bar{Q}_{j}^{\prime} \cap P\left(Q_{j}\right)^{-1}$, contradicting the fact that $Q_{j} \in \Omega$. Therefore, we can conclude that $B \in \Omega$. By application of Zorn's lemma, the set $\Omega$ has a maximal element. Let $R^{*}$ be such an element.

First of all, notice that by maximality of $R^{*}: R^{*}=\overline{R^{* \prime}}$. Therefore, by Lemma $1, R^{*}$ is absolute time-consistent, impatient and transitive. Let us show that $R^{*}$ is complete. If on the contrary, $R^{*}$ is not complete, there exist elements $x^{\prime}, y^{\prime} \in X$ and time-instances $t^{\prime}, v^{\prime} \in T$ such that $\left(\left(x^{\prime}, t^{\prime}\right),\left(y^{\prime}, v^{\prime}\right)\right) \in N\left(R^{*}\right)$. Consider the relation:

$$
Q=\overline{R^{*} \cup\left\{\left(\left(x^{\prime}, t^{\prime}\right),\left(y^{\prime}, v^{\prime}\right)\right)\right\}} .
$$

From Lemma 1, this relation is time-consistent, impatient and transitive, hence $\bar{Q}^{\prime} \cap P(Q)^{-1}=$ $\emptyset$. Moreover, it is strictly larger than $R^{*}$, i.e. $R^{*} \subset Q$. To show that $Q$ is in $\Omega$, we need to show that $Q$ extends $R$. That $R \subseteq Q$ is straightforward. Now assume on the contrary that $((y, v),(x, t)) \in P(R)$ and $((x, t),(y, v)) \in Q$. Consequently there is a sequence $x=x_{1}, \ldots, x_{n}=y$ in $X$ and sequences $t_{1}, \ldots, t_{n-1}$ and $v_{1}, \ldots, v_{n-1}$ in $T$ such that for all $i=1, . ., n-1$ :

$$
\left(\left(x_{i}, t_{i}\right),\left(x_{i+1}, v_{i}\right)\right) \in R^{*} \cup\left\{\left(\left(x^{\prime}, t^{\prime}\right),\left(y^{\prime}, v^{\prime}\right)\right)\right\}
$$

and

$$
t-v=\sum_{i=1}^{n-1}\left(t_{i}-v_{i}\right) .
$$

As $((x, t),(y, v)) \notin R^{*}\left(R^{*}\right.$ is an extension of $\left.R\right)$, there must be at least one instance of $i$ for which $\left(\left(x_{i}, t_{i}\right),\left(x_{i+1}, v_{i}\right)\right)=\left(\left(x^{\prime}, t^{\prime}\right),\left(y^{\prime}, v^{\prime}\right)\right)$. Let $L \subseteq\{1, \ldots, n-1\}$ be the set containing all these instances of $i$. We then have that:

$$
|L|\left(v^{\prime}-t^{\prime}\right)=\sum_{i \notin L}\left(t_{i}-v_{i}\right)+v-t .
$$

Introduce $t_{n}=v$ and $v_{n}=t$ an let us include the element $((y, v),(x, t))=\left(\left(x_{n}, t_{n}\right),\left(x_{1}, v_{n}\right)\right)$ into the sequence in order to make a loop joining $x_{n}=y$ back to $x_{1}=x$. We see that we can 
divide this loop into $|L|$ subsequences each starting with $y^{\prime}$ and ending with $x^{\prime}$. Denote the set of $i$ 's falling in the $l$-th sequence by $L_{l}$.

Consider elements $t_{l}$ and $v_{l}(l \in\{1, \ldots,|L|\}$ such that:

$$
v_{l}-t_{l}=\sum_{i \in L_{l}}\left(t_{i}-v_{i}\right)
$$

Observe, by absolute time-consistency of $R^{*}$, that for all $l \in L$, we have that $\left(\left(y^{\prime}, v_{l}\right),\left(x^{\prime}, t_{l}\right)\right) \in$ $R^{*}$. Let $l^{\prime} \in L$ be such that:

$$
v_{l^{\prime}}-t_{l^{\prime}} \geq v_{l}-t_{l}
$$

for all $l \in L$.We have that $|L|\left(v^{\prime}-t^{\prime}\right)=\sum_{l \in L}\left(v_{l}-t_{l}\right) \leq|L|\left(v_{l^{\prime}}-t_{l^{\prime}}\right)$, hence $v^{\prime}-t^{\prime} \leq v_{l^{\prime}}-t_{l^{\prime}}$. Consider elements $w$ and $q$ in $T$ such that $w-q=v^{\prime}-t^{\prime}$ and $v_{l^{\prime}}-w \leq t_{l^{\prime}}$. Such elements always exist. By absolute time consistency:

$$
\left(\left(y^{\prime}, v_{l^{\prime}}-v_{l^{\prime}}+w\right),\left(x^{\prime}, t_{l^{\prime}}-v_{l^{\prime}}+w\right)\right) \in R^{*} .
$$

By impatience of $R^{*}$, the fact that $t_{l^{\prime}}-v_{l^{\prime}}+w \leq q$ and transitivity of $R^{*}$, we derive that

$$
\left(\left(y^{\prime}, w\right),\left(x^{\prime}, q\right)\right) \in R^{*} .
$$

Using absolute time-consistency of $R^{*}$ a final time, we can conclude that

$$
\left(\left(x^{\prime}, v^{\prime}\right)\left(y^{\prime}, t^{\prime}\right)\right) \in R^{*}
$$

This is in contradiction with the assumption that $\left(\left(x^{\prime}, v^{\prime}\right),\left(y^{\prime}, t^{\prime}\right)\right) \in N\left(R^{*}\right)$. Conclude that $Q$ is in $\Omega$. As $R^{*} \subset Q$, this implies that $R^{*}$ is not maximal, again a contradiction. Conclude that $R^{*}$ is complete.

Now, we present the main part of the proof of theorem 1.

Proof. Let $R^{*}$ be a rationalization of $C$ and assume on the contrary that $((x, t),(y, v)) \in \bar{R}_{v}^{\prime}$ and $((y, v),(x, t)) \in P_{v}$. As $R^{*}$ is absolute time-consistent, transitive and impatient, we see from the definition of $R_{v}$ and Lemma 1 that $\bar{R}_{v}^{\prime} \subseteq R^{*}$. Also from the definition of $P_{v}$, we can conclude that $P_{v} \subseteq P\left(R^{*}\right)$. From this, we have that $((x, t),(y, v)) \in R^{*}$ and $((y, v),(x, t)) \in P\left(R^{*}\right)$, a contradiction.

To see the converse, let $C$ satisfy $A T A R P$, i.e. $\bar{R}_{v}^{\prime} \cap P_{v}^{-1}=\emptyset$. First we show that $P_{v}=$ $P\left(R_{v}\right)$. To see $P_{v} \subseteq P\left(R_{v}\right)$, observe that from the definitions $P_{v} \subseteq R_{v}$. If on the contrary, $((x, t),(y, v)) \in P_{v}$ and $((y, v),(x, t)) \in R_{v}$, we have a contradiction with $\bar{R}_{v}^{\prime} \cap P_{v}^{-1}=\emptyset$, hence $P_{v} \subseteq P\left(R_{v}\right)$. To see that $P\left(R_{v}\right) \subseteq P_{v}$, assume that $((x, t),(y, v)) \in P\left(R_{v}\right)$. Then, there is an $A \in \Sigma$ such that $(x, t) \in C(A)$ and $(y, v) \in A$. If on the contrary $((x, t),(y, v)) \notin P_{v}$, we must have that $(y, v) \in C(A)$. This implies that $((y, v),(x, t)) \in R_{v}$, a contradiction. Conclude that $P\left(R_{v}\right)=P_{v}$.

Now, we can rewrite $\bar{R}_{v}^{\prime} \cap P_{v}^{-1}=\emptyset$ as $\bar{R}_{v}^{\prime} \cap P\left(R_{v}\right)=\emptyset$. From Lemma 2, we derive that $R_{v}$ has a time-consistent and impatient ordering extension $R^{*}$. If $(x, t) \in C(A)$, we immediately have that $((x, t),(y, v)) \in R_{v}$ for all $(y, v) \in A$, hence $((x, t),(y, v)) \in R^{*}$ for all $(y, v) \in A$. If $(x, t) \notin C(A)$, we see from the non-emptiness ${ }^{4}$ of $C(A)$ that there is an $(y, v) \in A$ such that

\footnotetext{
${ }^{4}$ This follows from the assumption that the set $\Lambda$ does not contain the empty set.
} 
$((y, v),(x, t)) \in P_{v}=P\left(R_{v}\right)$. As $R^{*}$ is an extension of $R_{v}$, we derive that $((y, v),(x, t)) \in$ $P\left(R^{*}\right)$. Conclude that

$$
C(A)=\left\{(x, t) \in A \mid \forall(y, v) \in A,((x, t),(y, v)) \in R^{*}\right\} .
$$

Hence, $R^{*}$ is an absolute time-consistent and impatient rationlization of $C$.

\section{References}

Angeletos, Laibson, and Repetto. "The Hyperbolic Consumption Model: Calibration, Simulation, and Empirical Evaluation." Journal of Economic Perspectives 15: (2001) 47-68.

Debreu, Gerard. "Representation of a Preference Ordering by a Numerical Function." In: Thrall R, Coombs C and Davies $R$ (Eds.), Decision Processes. Wiley, New York.

Donaldson, David, and John A. Weymark. "A quasiordering is the intersection of orderings." Journal of Economic Theory 78: (1998) 382-387.

Duggan, John. "A general extension theorem for binary relations." Journal of Economic Theory 86: (1999) 1-16.

Durieu, J, H Haller, N Querou, and P Solal. "Ordinal games." www.awi.uniheidelberg.de/with2/seminar/SSO6_Abstract_Papers/Haller_HJNPOrdG\%.pdf .

Eisenhauer, Joseph. G, and Luigi Ventura. "The prevalence of hyperbolic discounting: some European evidence." Applied Economics 38: (2006) 1223-1234.

Fishburn, and Rubinstein. "Time preference." International Economic Review 23: (1982) 677-694.

Frederick, Shane, George Loewenstein, and Ted O'Donoghue. "Time discounting and time preference: a critical review." Journal of Economic Literature 40: (2002) 351-401.

Harvey. "Value functions for infinite-period planning." Management Science 32: (1986) $1123-1139$.

Richter, Marcel K. "Revealed preference theory." Econometrica 34: (1966) 635-645.

Sen, Amartya K. "Choice functions and revealed preference." Review of Economic Studies 38: (1971) 307-317.

Suzumura, Kotaro. "Remarks on the theory of collective choice." Economica 43: (1976) $381-390$

Szpilrajn, Edward. "Sur l'extension de l'ordre partiel." Fundamentae Mathematicae 16: (1930) 386-389. 